\title{
Coblator Assisted (Endoscopic) Recanalization as a Treatment Option for Laryngo -Tracheal Stenosis.
}

\author{
Jayasena K. D. N. U. D., Muhandiram M. E.C., Balasubramanium A., Daminda D.A.D.G.
}

\begin{abstract}
A49-year-old female with grade 3 tracheal stenosis underwent Coblator assisted recanalization and mytomycin-C application. Montgomery T-Tube was used to stent the lumen and she was successfully decanulated after one year. We believe that use of Coblator, mytomycin-C and $\mathrm{T}$ - tube resulted in successful outcome.
\end{abstract}

\section{Introduction}

Although many management options have been tried over the years, achieving complete recanalization of tracheal stenosis is still a challenge. We report the first case of a case series where a49-year-old female with tracheal stenosis was successfully decanulated after coblater assisted endoscopic recanalization.

\section{Case Report}

A 49 year old woman presented to ENT Unit, TH Anuradhapura with difficulty in breathing and stridor. Fiber optic nasolaryngoscopy (FONL) revealed an almost completely compromised airway at glottis and emergency tracheostomy was subsequently performed. She gave a history of intentional organophosphate poisoning, where she had been intubated for respiratory muscle paralysis and had been on prolonged ventilation in an ICU. Computed Tomography (CT) scan showed almost complete laryngotracheal stenosis (Grade 3) extending 25mm below vocal cords.

Coblator assisted recanalization was plannedas a long term treatment option. The procedure, it's outcome and the risks were explained and informed consent was obtained. Surgery was performed under general anaesthesia and she was ventilated through a cuffed tracheostomy tube during the surgery. The glottis was visualized from above using a rigid laryngoscope and endoscope. The stenotic area was recannalised by coblating residual scar tissue. A FONL light source was introduced just above the tracheostomy tube and it was used as a guide to prevent a false tract formation.

A mitomycin-C soaked gauze was applied to the coblated site for 3 minutes in order to minimize granulation tissue regrowth and a $12 \mathrm{G}$ Montgomery T-tube was placed to stent the recanalised site. After the $\mathrm{T}$ tube was inserted, the patient was ventilated through the $\mathrm{T}$ tube and a saline soaked gauze was placed at glottis to make a closed circuit for ventilation, which was removed at the end of the procedure.

One month later the patient readmitted complaining difficulty in breathing. FONL revealed a twisted T-tube and some granulation tissue formation. Granulation tissue was removed by coblation and the tube was repositioned under general anaesthesia.

The patient was trained to breathe through the glottis and to talk with closed T-tube which she learned quickly. After about 6 months she readmitted complaining of difficulty in breathing through the closed T tube. Although the glottis appeared normal, FONL through T-tube revealed some granulation tissue at the under surface of vocal cords obstructing the glottis. A third surgery was planned to remove the granulation tissue and the $\mathrm{T}$ tube orientation was reversed to prevent the upper limb touching the vocal cords.

The tube was kept for another 6 months. The patient was monitored monthly with FONL examination. After another 6 months the T tube was removed when the patient was comfortably breathing through glottis with closed T-tube. But a fenestrated tracheostomy tube was inserted after the surgery due to possible risk of tracheal collapse. FONL was performed 1 week later and tracheostomy was removed as there was 
adequate lumen to breathe. The patient is still under monthly follow up (Planned for 4 months). Currently the patient leads a normal life as she can breathe comfortably through nose with a reasonable speaking ability.

\section{Discussion}

Tracheal stenosis following prolonged intubation was first described in 1880 1. It's a serious but a rare complication of prolonged intubation. According to available data, incidence of symptomatic tracheal narrowing is $1 \%-2 \%$. And 4.6 cases per million per year 2,3 . The main identified reason is the loss of regional blood flow due to cuff pressure on the tracheal wall4.Ulceration of the mucosa, damage on cartilage and inflammatory reactions with associated granulation tissue leads to fibrous tissue formation, and contraction of fibrous scar tissue. (Medscape)

The severity is graded according to Cotton-Myer grading system,

- Grade $1-0-50 \%$ obstruction

- Grade 2 - 51-70\% obstruction

- Grade 3 - 71-99\% obstruction

- Grade 4 - No detectable lumen

Management options for tracheal stenosis include dilatation or airway reconstruction including laryngotracheoplasty(LTP), Cricotracheal resection(CTR), and tracheal resection(TR).

Patients undergoing dilation for laryngotracheal stenosis require multiple procedures.Major reconstructive procedures are well tolerated and currently represent a viable primary treatment for laryngotracheal stenosis5 but, this requires specialist centres to get favourable outcomes.

The endoscope assisted coblation surgery is a new minimally invasive method for treating subglottic stenosis. It can also be used to treat relapses6.
Coblation technology is based on a controlled, non-heat driven process that uses radiofrequency (RF) energy to excite the electrolytes in a conductive medium such as saline solution to create precisely focused plasma. Energized particles or ions in the plasma have sufficient energy to break, or dissociate molecular bonds within soft tissue at relatively low temperatures, typically 40 to $700 \mathrm{C}$. There for the damage on surrounding structures is less.

Granulation tissue formation following recanalization is a known complication and rarely causes complete airway obstruction. The time course is usually days to weeks after surgery.

Mitomycin is used as a chemotheraputic agent. Topical application of mitomycin can play an effective role in the prevention and treatment of scar formation in the aerodigestive tract 7 .

When granulation tissue becomes severe and airway obstruction is a concern, insertion of a t-tube should be considered 8. Montgomery T-tube is a silicone stent for the trachea with an external limb. This stent provides airway support for patients with a tracheal stenosis to allow the trachea to heal and avoids the need for a long term tracheostomy tube. Patients can wear closed T-tubes from 1-12 years 9 .

In our patient, we believe, use of coblator minimized surrounding tissue damage and application of mytomycin kept growth of granulation tissue to a very minimum and keeping the patient on a $\mathrm{T}$ tube allowed adequate time for the tracheal mucosa to regrow resulting in less chance of restenosis.

\section{Key messages}

This is the first successful decantation of a series of similar cases and Coblation appears to be a promising technique in recanalization of tracheal stenosis however, further quality studies are recommended to assess its effectiveness. 


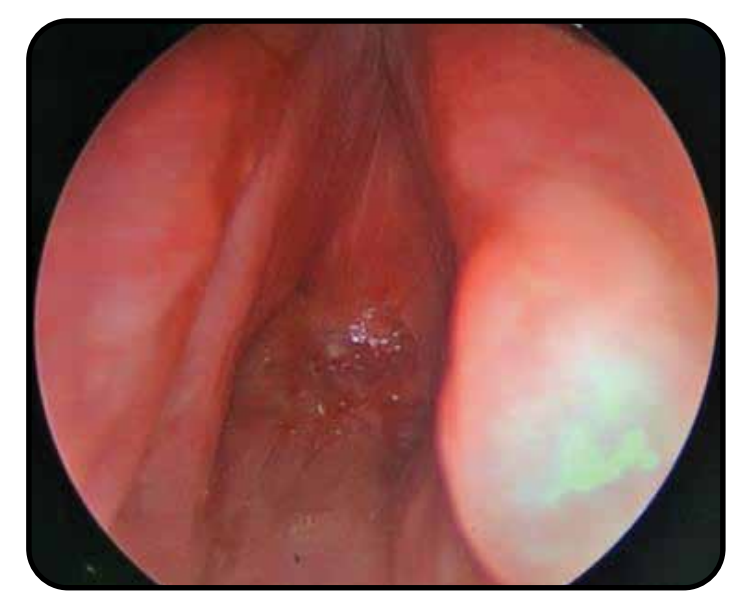

Fig 1 - FONL view of Glottis at presentation

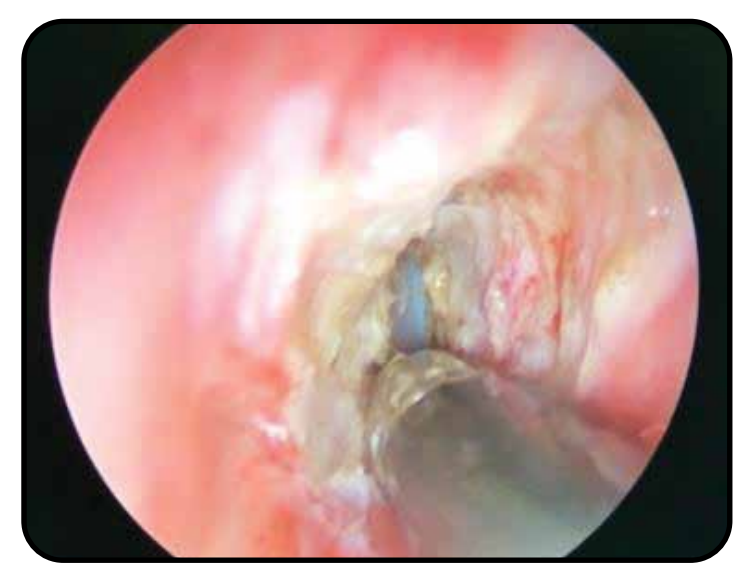

Fig 2 - Coblating the stenosed area

\section{References}

1. MacEwen W. Clinical Observations on the Introduction of Tracheal Tubes by the Mouth Instead of Performing Tracheotomy or Laryngotomy. Br Med J. 1880, 2: 122-124.

2. Kastanos N, Estopa Miro R, Marin Perez A, Xaubet Mir A, Agusti-Vidal A.Laryngotracheal injury due to endotracheal intubation: incidence, evolution, and predisposing factors. A prospective longterm study. Critical care medicine. 1983, 11 (5): 362-367. 10.1097/00003246198305000-00009
3. Nouraei SA, Ma E, Patel A, Howard DJ, Sandhu GS: Estimating the population incidence of adult post-intubation laryngotracheal stenosis. Clin Otolaryngol. 2007, 32 (5): 411-412.

4. Weymuller EA. Laryngeal injury from prolonged endotracheal intubation. The Laryngoscope. 1988, 98 (8 Pt 2 Suppl 45): $1-15$

5. Herrington, H. C., Weber, S. M. and Andersen, P. E. (2006). Modern Management of Laryngotracheal Stenosis. The Laryngoscope, 116: 1553-1557. doi:10.1097/01.mlg.0000228006.21941.12

6. Lin Chung Er Bi Yan HouTou Jing WaiKeZaZhi. 2013 Jun;27(11):587-9.

7. Rahbar R, Jones DT, Nuss RC, Roberson DW, Kenna MA, McGill TJ, Healy GB. The Role of Mitomycin in the Prevention and Treatment of Scar Formation in the PediatricAerodigestiveTractFriend or Foe?. Arch Otolaryngol Head Neck Surg. 2002;128(4):401-406. doi:10.1001/ archotol.128.4.40

8. Hugh G, Auchincloss, Cameron D: Complications after tracheal resection and reconstruction: prevention and treatment.2016 Mar; 8(Suppl 2): S160 S167.10.3978/j.issn.2072-1439.2016.01.86 PMCID: PMC4775259

9. Morshed K, Szymański M, Gołabek W: The use of tracheostomy T-tube in the treatment of tracheal stenosis.Otolaryngol Pol. 2005;59(3):361-4. 